This item was submitted to Loughborough's Research Repository by the author.

Items in Figshare are protected by copyright, with all rights reserved, unless otherwise indicated.

\title{
Disturbance rejection for nonlinear uncertain systems with output measurement errors: Application to a helicopter model
}

\section{PLEASE CITE THE PUBLISHED VERSION}

https://doi.org/10.1109/TII.2019.2910841

\section{PUBLISHER}

Institute of Electrical and Electronics Engineers (IEEE)

VERSION

AM (Accepted Manuscript)

\section{LICENCE}

CC BY-NC-ND 4.0

\section{REPOSITORY RECORD}

Yan, Yunda, Chuanlin Zhang, Cunjia Liu, Jun Yang, and Shihua Li. 2019. "Disturbance Rejection for Nonlinear Uncertain Systems with Output Measurement Errors: Application to a Helicopter Model". Loughborough University. https://hdl.handle.net/2134/37612. 


\title{
Disturbance Rejection for Nonlinear Uncertain Systems with Output Measurement Errors: Application to a Helicopter Model
}

\author{
Yunda Yan, Member, IEEE, Chuanlin Zhang, Member, IEEE, Cunjia Liu, Member, IEEE, \\ Jun Yang, Senior Member, IEEE, Shihua Li, Senior Member, IEEE
}

\begin{abstract}
As a virtual sensor, disturbance observer provides an alternative approach to reconstruct lumped disturbances (including external disturbances and system uncertainties) based upon system states/outputs measured by physical sensors. Not surprisingly, measurement errors bring adverse effects on the control performance and even the stability of the closed-loop system. Toward this end, this paper investigates the problem of disturbance observer based control for a class of disturbed uncertain nonlinear systems in the presence of unknown output measurement errors. Instead of inheriting from the estimation-error-driven structure of Luenberger type observer, the proposed disturbance observer only explicitly uses the control input. It has been proved that the proposed method endows the closed-loop system with strong robustness against output measurement errors and system uncertainties. With rigorous analysis under the semiglobal stability criterion, the guideline of gain choice based upon the proposed structure is provided. To better demonstrate feature and validity of the proposed method, numerical simulation and comparative experiments of a helicopter model are implemented.
\end{abstract}

Index Terms-Disturbance rejection, disturbance observer, output measurement error, robust control, semiglobal stability.

\section{INTRODUCTION}

$\mathbf{H}$ OW to attenuate external disturbances and system uncertainties has always been an attractive subject in both control theory and practical engineering [1]. Targeted at this old but still open issue, disturbance observer based control (DOBC) method, initiatively put forward by Kouhei Ohnishi [2] and independently developed by others [3]-[5], provides an alternative practical solution and has also proved its effectiveness in plenty of industrial sites, e.g., robots [6]-

This work was supported in part by the National Natural Science Foundation of China under the Grants 61473080, 61573099, 61750110525, and 61633003 , in part by The Program for Professor of Special Appointment (Eastern Scholar) at Shanghai Institutions of Higher Learning, in part by the UK Engineering and Physical Science Research Council (EPSRC) under the Grant EP/P012868/1. (Corresponding author: Shihua Li.)

Y. Yan, J. Yang, and S. Li are with the Key Laboratory of Measurement and Control of CSE, Ministry of Education, School of Automation, Southeast University, Nanjing 210096, China (e-mail: yyd@seu.edu.cn, j.yang84@seu.edu.cn and Ish@seu.edu.cn).

C. Zhang is with the College of Automation Engineering, Shanghai University of Electric Power, Shanghai 200090, China (email: clzhang@shiep.edu.cn).

C. Liu is with the Department of Aeronautical and Automotive Engineering, Loughborough University, Loughborough LE11 3TU, U.K. (e-mail: c.liu5@lboro.ac.uk).
[8], motion control systems [9]-[12] and power electronics [13], [14].

In addition to those successful real-life applications, several modified DOBC methods have also been developed from different theoretical aspects. 1) Integrating Multiple Disturbance Models: Inspired by the internal model principle [15], appropriately utilizing different disturbance models, DOBC methods for nonlinear systems subject to polynomial disturbances are proposed in [16], [17] and subject to periodic disturbances with known and fixed frequencies are proposed in [18] and with unknown time-varying frequencies are proposed in [19]. Besides, these DOBC methods have already been applied in motion control systems to suppress ripples in torque/speed/position [9], [20]. 2) Faster Convergences and Higher-Precision: Instead of focusing on disturbance modelling, more advanced convergence properties of disturbance observer itself is investigated. Inspired by the higher-order sliding mode method [21], a finite-time disturbance observer is proposed in [22], [23] for integral chain systems with bounded disturbances. Furthermore, to solve the difficulties in parameter selection of sliding mode disturbance observer, a homogeneous disturbance observer is proposed in [24], whose gain tuning mechanism is similar with that of the classic linear DOBC method. 3) Other Improvements: Considering the restrictions on communication resources in the networked environment, the event-triggered DOBC methods are proposed for disturbed systems in [25], [26]. To suppress the effect of sensor noise, a modified DOBC method is proposed in [27]. In [28], adaptive gains of disturbance observer are designed to achieve the linear minimum variance estimation for both states and total disturbance. In [29], composite learning based DOBC methods are proposed for disturbed uncertain systems. In [30], an active disturbance rejection adaptive controller is proposed for tracking control of nonlinear systems with parametric uncertainties and uncertain nonlinearities. From the classic forms to these modified ones, the basic idea of DOBC methods is the same, i.e., by treating disturbances as system states, then design an observer for the extended system and a controller including a compensation part for disturbances. Besides, to a large extent, the disturbance observer mechanism is inherited from the Luenberger type observer (see, e.g., the classic reduced-/full-order ones [3], [4], the homogeneous one [24], the event-triggered ones [25], [26] and references therein), whose estimation precision directly depends on the accuracy of system states/outputs measured by various kinds 
of physical sensors.

However, there are many physical factors that affect the measurement accuracy. On one hand, due to improper calibration of measurement instruments or unpredictable changes of environment, measurement errors widely exist in industrial sensors [31]. Taking potentiometer as an example, which is a three-terminal resistor and is always used in displacement measurement, its precision is easily affected by environment temperature and its allowable sensitivity error even could be $\pm 20 \%$ according to different grades [32]. On the other hand, even if sensor accuracy is high enough, measurement error would appear in several special scenarios. For instance, in the visual servo problem of aircraft, the coordinate of the target is acquired by the image from camera, which is directly related with the height of aircraft [33]. However, even in the case of hover flight control, its height inevitably fluctuates due to aerodynamic characteristics, leading to the measurement errors in the position of target. Notably, the adverse effect of measurement errors is quite challenging to be tackled, not only because accurate information of system states/outputs is fundamental in the design of DOBC methods, but also because only a rough range of this kind of error, rather than an exact function to describe this, is available in most industrial situations.

Motivated by the above-mentioned challenging issues, a new DOBC method is proposed in this paper for a class of uncertain nonlinear systems with both external disturbances and output measurement errors. Compared with the previous related results, the main contributions can be summarized by the following two aspects.

1) Disturbance rejection considering output measurement errors. As an inheritance and development of the conventional active disturbance rejection control (ADRC) method [4], [34]-[36], we present the first attempt on estimation and attenuation of disturbances/uncertainties to deal with the adverse effect caused by output measurement errors. To be more specific, inspired by pioneer works focusing on output measurement errors [37], [38], this problem is solved by reducing the use of the measured output and introducing a non-Luenberger type disturbance observer.

2) Semiglobal stabilization based bandwidth specification. Under a more practical control objective, namely, semiglobal control objective [39]-[41], the proposed method is able to deal with more general system nonlinearities and explicitly provides the guideline of choosing the bandwidths of both the controller and disturbance observer, which is directly related to the ranges of the initial conditions, system nonlinearities and allowable sensitivity errors. Besides, disturbance rejection ability against its model error is quantitatively analyzed, based upon which the stabilization precisions could be further increased.

The remainder of this paper is organized as follows. The problem formulation is provided in Section II. The new DOBC structure with its corresponding gain guideline based upon the semiglobal stabilization analysis is introduced in Section III.
Robust elevation angle control results of a helicopter model under the conventional proportional-integral-derivative (PID) method, the linear ADRC method and the proposed method are provided in Section IV. Finally, Section V concludes this paper.

Notations: Throughout the paper, symbols $\mathbb{R}, \mathbb{N}, \mathbb{R}^{+}, \mathbb{N}^{+}$ and $\mathbb{C}^{0}$ denote the real number set, the natural number set, the positive number set, the positive integer number set and the set of all continuous functions, respectively. $\forall x \in \mathbb{R}^{n},\|x\| \triangleq$ $\sqrt{x^{\top} x}$. Besides, let $\mathbb{N}_{i: j} \triangleq\{i, i+1, \cdots, j\}, \forall i, j \in \mathbb{N}$ and $i \leq j$.

\section{Problem Formulation}

This paper considers the problem of semiglobal output feedback stabilization for a class of uncertain nonlinear systems with both external disturbances and output measurement errors in the following form:

$$
\left\{\begin{aligned}
\dot{x}_{i} & =x_{i+1}+f_{i}(t, x), \quad i \in \mathbb{N}_{1: n-1} \\
\dot{x}_{n} & =u+f_{n}(t, x)+d(t) \\
y & =x_{1} \\
y_{m} & =\theta(t) y
\end{aligned}\right.
$$

where $x \triangleq \operatorname{col}\left(x_{1}, \cdots, x_{n}\right) \in \mathbb{R}^{n}, u \in \mathbb{R}, y \in \mathbb{R}, y_{m} \in \mathbb{R}, d(t) \in \mathbb{R}$, $f_{i}(t, x) \in \mathbb{R}, i \in \mathbb{N}_{1: n}$ and $\theta(t) \in \mathbb{R}$ are the system state, control input, system output, measured output, external disturbance, system uncertainties and sensor sensitivity, respectively.

To begin with, following assumptions are required.

Assumption 1: $\theta(t) \in \mathbb{C}^{0}$ is unknown, strictly positive and bounded.

Assumption 2: For each nonlinear function $f_{i}(t, x)$, there exists a $\mathbb{C}^{0}$ function $g_{i}: \mathbb{R} \longmapsto \mathbb{R}^{+}$such that

$$
\left|f_{i}(t, x)\right| \leq g_{i}(y)\left(\left|x_{1}\right|+\cdots+\left|x_{i}\right|\right), \quad i \in \mathbb{N}_{1: n} .
$$

Assumption 3: The external disturbance can be expressed by the following form:

$$
d(t)=a_{1}+a_{2} t+\cdots+a_{m} t^{m-1}+r(t), m \in \mathbb{N}^{+}
$$

where $a_{i} \in \mathbb{R}, i \in \mathbb{N}_{1: m}$ are unknown constants and $r(t)$ is a $m$-th differentiable unknown residual term and there exists a constant $\bar{r} \geq 0$ such that $\left|r^{(m)}(t)\right| \leq \bar{r}$.

Following explanations are provided for the considered output measurement error, system uncertainties and external disturbance.

Remark 1: Industrial measurement error can be classified as random error and systematic error whilst the later can also be subdivided as zero setting error and scale factor error [31], [42]. Random error often has a Gaussian normal distribution and is usually handled by Kalman filter in practices. Besides, random error is also considered in the design of high gain state observer [43]-[47]. Recalibrating sensor will reduce or even eliminate zero setting error. As for the scale factor error, i.e., $y_{m}=\theta(t) y$, it is always generated by unpredictable changes in measurement, and hence, is impossible to acquire a precise sensor sensitivity function $\theta(t)$. For engineers, however, it is still possible to give a rough range of $\theta(t)$ based upon their experience on sensor qualities or working conditions.

Remark 2: Compared with the conventional DOBC methods considering system uncertainties [5], [10], [48], [49], here we 
further extend the results to non-Lipschitz cases, as presented in Assumption 2, which is also much weaker than the linear growth condition adopted in [38]. The generalized condition of Assumption 2 is introduced and detailedly explained in [41] for nonlinear control design. In this paper, under a more practical control objective, namely, semiglobal control objective [39]-[41], we will show that Assumption 2 will be sufficient to solve the robust stabilization problem of uncertain system (1) with both external disturbances and output measurement errors.

Remark 3: The considered external disturbance (3) is regarded as a Taylor series expansion with respect to time [16], [17]. When $r(t)=0$ and $m=1$ or 2, disturbance (3) is a constant or ramp one, which widely exists in motion control systems as load torque [11], [20]. Different from the exact disturbance models used in [3], [16], [18], [48], the non-vanishing uncertainty $r(t)$ in (3) represents its model error, which brings convenience to the quantitative analysis of disturbance rejection ability [see Section III-B for details].

\section{Main Results}

To solve the above-mentioned problem, we firstly present a new DOBC structure. Secondly, based upon the proposed structure and with rigorous analysis under the semiglobal stability criterion, the guideline of subtly choosing gains of both disturbance observer and composite controller is given step-by-step. A numerical simulation ends this section to show the validity of the proposed method on disturbance estimation and attenuation.

\section{A. Composite Control Strategy}

1) Disturbance Observer Design: Following the basic idea of the conventional disturbance observer design, by letting $x_{n+i} \triangleq d^{(i-1)}, i \in \mathbb{N}_{1: m}$, system (1) is then extended as follows:

$$
\left\{\begin{aligned}
\dot{x}_{i} & =x_{i+1}+f_{i}(t, x), \quad i \in \mathbb{N}_{1: n-1} \\
\dot{x}_{n} & =u+f_{n}(t, x)+x_{n+1} \\
\dot{x}_{j} & =x_{j+1}, \quad j \in \mathbb{N}_{n+1: n+m-1} \\
\dot{x}_{n+m} & =r^{(m)}(t) \\
y_{m} & =\theta(t) x_{1} .
\end{aligned}\right.
$$

Different from the conventional one inherited from Luenberger type, we construct the following disturbance observer for the extended system (4):

$$
\left\{\begin{aligned}
\dot{\hat{x}}_{i} & =\hat{x}_{i+1}-h_{i} \hat{x}_{1}, \quad i \in \mathbb{N}_{1: n-1} \\
\dot{\hat{x}}_{n} & =u+\hat{x}_{n+1}-h_{n} \hat{x}_{1} \\
\dot{\hat{x}}_{j} & =\hat{x}_{j+1}-h_{j} \hat{x}_{1}, j \in \mathbb{N}_{n+1: n+m-1} \\
\dot{\hat{x}}_{n+m} & =-h_{n+m} \hat{x}_{1}
\end{aligned}\right.
$$

where $\hat{x}_{i}$ are the estimate of $x_{i}, h_{i} \triangleq \ell_{o}^{i} \tilde{h}_{i}, i \in \mathbb{N}_{1: n+m}, \ell_{o} \geq 1$ is a constant parameter which will be specified later and $\tilde{h}_{i}$ are coefficients of Hurwitz polynomial $h_{o}(s)=s^{n+m}+\sum_{i=1}^{n+m} \tilde{h}_{i} s^{n+m-i}$.

Let $\tilde{e}_{i} \triangleq e_{i} / \ell_{o}^{i-1}, i \in \mathbb{N}_{1: n+m}$, where $e_{i} \triangleq x_{i}-\hat{x}_{i}$. Noting that $\hat{x}_{1}=x_{1}-\tilde{e}_{1}$, the following estimation error system is obtained:

$$
\dot{\tilde{e}}=\ell_{o} A_{o} \tilde{e}+\ell_{o} H_{o} x_{1}+F
$$

where

$$
\begin{aligned}
& \tilde{e} \triangleq \operatorname{col}\left(\tilde{e}_{1}, \cdots, \tilde{e}_{n+m}\right), H_{o} \triangleq \operatorname{col}\left(\tilde{h}_{1}, \cdots, \tilde{h}_{n+m}\right) \\
& F \triangleq \operatorname{col}\left(f_{1}, \cdots, \frac{f_{n}}{\ell_{o}^{n-1}}, 0, \cdots, 0, \frac{r^{(m)}}{\ell_{o}^{n+m-1}}\right) \\
& A_{o} \triangleq\left(\begin{array}{cccc}
-\tilde{h}_{1} & 1 & \cdots & 0 \\
\vdots & \vdots & \ddots & \vdots \\
-\tilde{h}_{n+m-1} & 0 & \cdots & 1 \\
-\tilde{h}_{n+m} & 0 & \cdots & 0
\end{array}\right) .
\end{aligned}
$$

2) Composite Controller Design: With the assist of the estimates, we design the following composite controller:

$$
u=-k_{n} y_{m}-k_{n-1} \hat{x}_{2}-\cdots-k_{1} \hat{x}_{n}-\hat{x}_{n+1}
$$

where $k_{i} \triangleq\left(\ell_{o} \ell_{c}\right)^{i} \tilde{k}_{i}, i \in \mathbb{N}_{1: n}, \ell_{c} \geq 1$ is a scaling gain whose guideline will be made precise in the next subsection and $\tilde{k}_{i}$ are coefficients of Hurwitz polynomial $h_{c}(s)=s^{n}+\sum_{i=1}^{n} \tilde{k}_{i} s^{n-i}$.

Letting $\tilde{x}_{1} \triangleq x_{1}, \quad \tilde{x}_{i} \triangleq \hat{x}_{i} /\left(\ell_{o} \ell_{c}\right)^{i-1}, i \in \mathbb{N}_{2: n}$ and $\tilde{u} \triangleq(u+$ $\left.\hat{x}_{n+1}\right) /\left(\ell_{o} \ell_{c}\right)^{n}$, with $\tilde{x}_{1}=\hat{x}_{1}+\tilde{e}_{1}$ in mind, a direct calculation leads to the following system:

$$
\begin{aligned}
\dot{\tilde{x}}= & \ell_{o} \ell_{c} A_{c} \tilde{x}+\frac{\ell_{o}}{\ell_{c}} H_{c}\left(\tilde{x}_{1}-\tilde{e}_{1}\right)+I_{1}\left(\ell_{o} \tilde{e}_{2}+f_{1}(t, x)\right) \\
& +I_{2} \ell_{o} \ell_{c} \tilde{k}_{n}(1-\theta(t)) \tilde{x}_{1}
\end{aligned}
$$

where

$$
\begin{aligned}
\tilde{x} & \triangleq \operatorname{col}\left(\tilde{x}_{1}, \cdots, \tilde{x}_{n}\right) \\
H_{c} & \triangleq \operatorname{col}\left(0,-\tilde{h}_{2}, \cdots,-\frac{\tilde{h}_{n}}{\ell_{c}^{n-2}}\right) \\
I_{1} & \triangleq \operatorname{col}(1,0, \cdots, 0), I_{2} \triangleq \operatorname{col}(0, \cdots, 0,1) \\
A_{c} & \triangleq\left(\begin{array}{cccc}
0 & 1 & \cdots & 0 \\
\vdots & \vdots & \ddots & \vdots \\
0 & 0 & \cdots & 1 \\
-\tilde{k}_{n} & -\tilde{k}_{n-1} & \cdots & -\tilde{k}_{1}
\end{array}\right)
\end{aligned}
$$

\section{B. Semiglobal Stability Analysis}

Theorem 1: Under Assumptions 1 to 3, consider the closedloop system, including (1), (5) and (7), with an allowable sensitivity error $\Delta$ such that $\theta(t) \in[1-\Delta, 1+\Delta]$. The following two statements hold:

1) All the trajectory starting from a compact set will be uniformly bounded.

2) When $\bar{r}=0$, the closed-loop system is semiglobally stable; otherwise, is semiglobally practically stable ${ }^{1}$.

Proof: For orderness, we break up the process of proof into the following three parts.

1) Preliminaries: Since that $A_{o}$ and $A_{c}$ are both Hurwitz, there exist two positive definite matrixes $P_{o} \in \mathbb{R}^{(n+m) \times(n+m)}$ and $P_{c} \in \mathbb{R}^{n \times n}$ such that $A_{o}^{\top} P_{o}+P_{o} A_{o}=-I_{o}$ and $A_{c}^{\top} P_{c}+P_{c} A_{c}=-I_{c}$, where $I_{o} \in \mathbb{R}^{(n+m) \times(n+m)}$ and $I_{c} \in \mathbb{R}^{n \times n}$ are identity matrixes. Thus, construct two Lyapunov functions as $V_{o}(\tilde{e}) \triangleq \tilde{e}^{\top} P_{o} \tilde{e}$ and $V_{c}(\tilde{x}) \triangleq \tilde{x}^{\top} P_{c} \tilde{x}$ for systems (6) and (8), respectively.

For simplicity, following two propositions are given, whose proofs are collected in Appendixes B and C, respectively.

\footnotetext{
${ }^{1}$ The definitions of semiglobal stability and semiglobal practical stability are provided in Appendix $A$ for the convenience of the readers.
} 
Proposition 1: There exist constants $c_{i}>0, i \in \mathbb{N}_{1: 2}$ such that

$$
\begin{aligned}
\left.\dot{V}_{o}(\tilde{e})\right|_{(6)} \leq & -\left(\frac{\ell_{o}}{2}-c_{1}-2 n g^{2}(y)\right)\|\tilde{e}\|^{2} \\
& +\left(c_{2} \ell_{o}+2 n g^{2}(y) \ell_{c}^{2 n-2}\right)\|\tilde{x}\|^{2}+\frac{\bar{r}^{2}}{\ell_{o}^{2(n+m-1)}} .
\end{aligned}
$$

Proposition 2: There exist constants $c_{i}>0, i \in \mathbb{N}_{3: 5}$ and $c^{*}>0$ such that

$$
\left.\dot{V}_{c}(\tilde{x})\right|_{(8)} \leq-c^{*} \ell_{o} \ell_{c}\|\tilde{x}\|^{2}+\left(c_{5} \ell_{o}+2 \lambda_{c} g(y)\right)\|\tilde{x}\|^{2}+\frac{\ell_{o}}{4}\|\tilde{e}\|^{2}
$$

where $\lambda_{c} \triangleq \lambda_{\max }\left(P_{c}\right)$.

Let $V(\tilde{e}, \tilde{x}) \triangleq V_{o}(\tilde{e})+V_{c}(\tilde{x})$. In view of (9) and (10), we then have

$$
\begin{aligned}
\left.\dot{V}(\tilde{e}, \tilde{x})\right|_{(6)-(8)} \leq & -\left(\frac{\ell_{o}}{4}-c_{1}-2 n g^{2}(y)\right)\|\tilde{e}\|^{2}-\ell_{o} \ell_{c}\left(c^{*}-\frac{c_{2}+c_{5}}{\ell_{c}}\right. \\
& \left.-\frac{2 n g^{2}(y) \ell_{c}^{2 n-3}}{\ell_{o}}-\frac{2 \lambda_{c} g(y)}{\ell_{o} \ell_{c}}\right)\|\tilde{x}\|^{2}+\frac{\bar{r}^{2}}{\ell_{o}^{2(n+m-1)}} .
\end{aligned}
$$

2) Semiglobal Attractivity Analysis: For all initial states satisfying

$$
\operatorname{col}\left(x_{1}(0), \cdots, x_{n+m}(0), \hat{x}_{1}(0), \cdots, \hat{x}_{n+m}(0)\right) \in \Gamma \triangleq[-\rho, \rho]^{2 n+2 m}
$$

where $\rho>0$ is a constant that could be arbitrarily large, we define the following compact set:

$$
\Omega_{M} \triangleq\left\{\operatorname{col}\left(\tilde{e}_{1}, \cdots, \tilde{e}_{n+m}, \tilde{x}_{1}, \cdots, \tilde{x}_{n}\right) \mid V(\tilde{e}, \tilde{x}) \leq M\right\}
$$

where $M \triangleq \max _{\tilde{e}_{i} \in[-2 \rho, 2 \rho], \tilde{x} j \in[-\rho, \rho], i \in \mathbb{N}_{1: n+m}, j \in \mathbb{N}_{1: n}}\{V(\tilde{e}, \tilde{x})\}>0$. Noting that $y=\tilde{x}_{1}+\tilde{e}_{1}$ and $g(y) \in \mathbb{C}^{0}$, let $g^{*} \triangleq$ $\max _{y \in[-3 \rho, 3 \rho]}\{g(y)\}$. By subtly choosing $\ell_{c}$ and $\ell_{o}$ as

$$
\begin{gathered}
\ell_{c} \geq \max \left\{1, \frac{6\left(c_{2}+c_{5}\right)}{c^{*}}\right\} \triangleq \underline{\ell_{c}}(\Delta) \\
\ell_{o} \geq \max \left\{1,4\left(C+c_{1}+2 n g^{* 2}\right), \frac{12 n g^{* 2} \ell_{c}^{2 n-3}}{c^{*}}, \frac{12 \lambda_{c} g^{*}}{c^{*} \ell_{c}}, \frac{2 C}{c^{*} \ell_{c}},\right. \\
\left.\left(\frac{c_{6} C M}{4 \bar{r}^{2}}\right)^{2 n+2 m-2}\right\} \triangleq \underline{\ell_{o}}\left(\ell_{c}, \bar{r}, \Delta, C, M\right)
\end{gathered}
$$

where $C>0$ is the convergence rate that also could be arbitrarily large and $c_{6} \triangleq \min \left\{1 / \lambda_{\max }\left(P_{c}\right), 1 / \lambda_{\max }\left(P_{o}\right)\right\}$, we have

$$
\left.\dot{V}(\tilde{e}, \tilde{x})\right|_{\Omega_{M}} \leq-c_{6} C \cdot V(\tilde{e}, \tilde{x})+\frac{\bar{r}^{2}}{\ell_{o}^{2(n+m-1)}} .
$$

Notably, constants $c_{i}, i \in \mathbb{N}_{1: 6}$ and $c^{*}$ are only related with a priori constants $\tilde{h}_{i}, i \in \mathbb{N}_{1: n+m}$ and $\tilde{k}_{j}, j \in \mathbb{N}_{1: n}$, making sense of (14) and (15) [see Appendixes B and C for detailed definitions of $c_{i}, i \in \mathbb{N}_{1: 6}$ and $\left.c^{*}\right]$.

The main result of the attraction domain is presented as the following proposition, whose proof is left in Appendix D.

Proposition 3: For initial state $\operatorname{col}(\tilde{e}(0), \tilde{x}(0))$, starting from $\Omega_{M}$, its trajectory col $(\tilde{e}(t), \tilde{x}(t))$ will stay in $\Omega_{M}$ forever.
3) Local Convergence: With Proposition 3 in mind, one arrives at that $(12) \Rightarrow \operatorname{col}(\tilde{e}(0), \tilde{x}(0)) \in \Omega_{M} \Rightarrow \operatorname{col}(\tilde{e}(t), \tilde{x}(t))$ $\in \Omega_{M}, t \in[0, \infty)$. Solving the differential inequality (16) gives

$$
c_{6}\left(\|\tilde{e}\|^{2}+\|\tilde{x}\|^{2}\right) \leq V(t) \leq V(0) e^{-c_{6} C t}+\frac{\bar{r}^{2}-e^{-c_{6} C t}}{c_{6} C \ell_{o}^{2(n+m-1)}},
$$

which leads to $\|\tilde{e}(\infty)\|,\|\tilde{x}(\infty)\| \leq \bar{r} /\left(c_{6} \sqrt{C} \ell_{o}^{n+m-1}\right)$. Furthermore, the following four inferences hold:

$\left(A_{1}\right)$ Estimation errors $\left|e_{i}(\infty)\right| \leq \bar{r} /\left(c_{6} \sqrt{C} \ell_{o}^{n+m-i}\right), i \in \mathbb{N}_{1: n+m}$.

$\left(A_{2}\right)$ Estimated states $\left|\hat{x}_{j}(\infty)\right| \leq \bar{r} \ell_{c}^{j-1} /\left(c_{6} \sqrt{C} \ell_{o}^{n+m-j}\right), j \in \mathbb{N}_{2: n}$.

$\left(A_{3}\right)$ Output $|y(\infty)|=\left|x_{1}(\infty)\right| \leq \bar{r} /\left(c_{6} \sqrt{C} \ell_{o}^{n+m-1}\right)$.

$\left(A_{4}\right)$ System states $\left|x_{k}(\infty)\right| \leq\left|e_{k}(\infty)\right|+\left|\hat{x}_{k}(\infty)\right|, k \in \mathbb{N}_{1: n}$.

With $\left(A_{1}\right)$ to $\left(A_{4}\right)$, one gets that when $\bar{r}=0$, all the abovementioned variables are asymptotically stable; otherwise, can be rendered arbitrarily small by choosing a suitably large $C$.

This completes the proof of Theorem 1 .

Remark 4: As shown in Fig. 1, the proposed disturbance observer (5) is different from the most existing ones, i.e., the nonlinearities $f_{i}(t, x), i \in \mathbb{N}_{1: n}$ and the measurement output $y_{m}$ are not explicitly used for the disturbance observer design. The main reason for not using the estimation error based correction term in the disturbance observer design, i.e., $y_{m}-\theta(t) \hat{x}_{1}$, is that the accurate information of the sensor sensitivity $\theta(t)$ is unavailable.

Remark 5: In practical applications, the allowable sensitivity error $\Delta$ should be given a priori based upon engineers' experience and specific working conditions, as mentioned in Remark 1. After giving $\Delta$, with (22), the restriction for controller gains, i.e., $\widetilde{k}_{n} \lambda_{c}>1 /(2 \Delta)$, is determined. With (14) and (15) together, gains of both disturbance observer and composite controller are then specified.

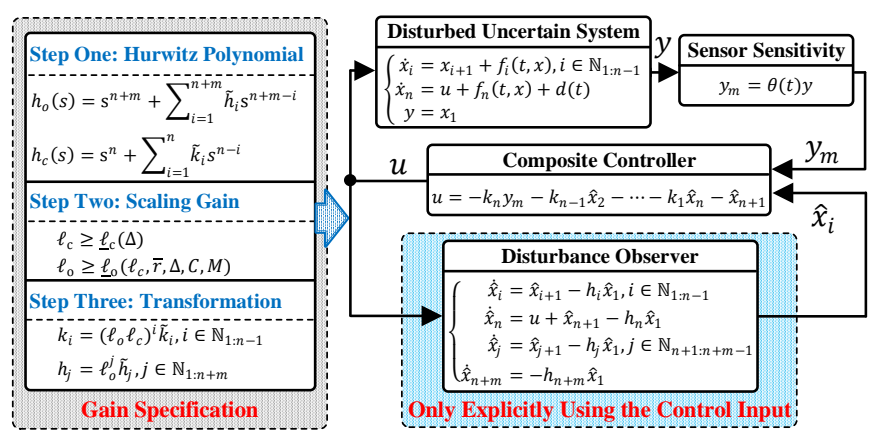

Figure 1. The implementation block diagram of the proposed method: NonLuenberger type structure and its corresponding gain specification.

\section{Numerical Simulation}

To show the semiglobal stabilization performance of the proposed DOBC method, a second-order system (1) with a sawtooth external disturbance is considered. In this numerical example, the system uncertainties are $f_{1}(t, x)=\left|x_{1}\right| \ln \left(1+x_{1}^{2}\right)$ and $f_{2}(t, x)=x_{1}\left(x_{1}+x_{2}\right)$, the external disturbance is depicted in the red dash line of Fig. 3 (b), the sensor sensitivity is $\theta(t)=1+0.2|\sin (20 t)|$ or $\theta(t)=1+0.4|\sin (40 t)|$, and the initial system state is $\left(x_{1}(0), x_{2}(0)\right)=(-0.1,0.1)$. 
Before implementing the proposed controller, it is worth noting that the nonlinearities $f_{1}(t, x)$ and $f_{2}(t, x)$ do not satisfy the linear growth condition in [38]. Besides, the exact feedback linearization based nonlinear control method [39] can also not be applied in this numerical example due to the ill-defined relative degree of $x_{1}$, even without output measurement error. In the simulation, the control parameters are chosen as $\ell_{c}=4$, $\ell_{o}=6,\left(\tilde{k}_{1}, \tilde{k}_{2}\right)=(6,9),\left(\tilde{h}_{1}, \tilde{h}_{2}, \tilde{h}_{3}, \tilde{h}_{4}\right)=(8,24,32,16)$ and the initial system state of the proposed disturbance observer is $\left(\hat{x}_{1}(0), \hat{x}_{2}(0), \hat{x}_{3}(0), \hat{x}_{4}(0)\right)=(0,-3,-10,0)$.

As shown in Fig. 2 (c), the stabilization objective of system states is realized by the proposed controller. The curves of the measured outputs are presented in Fig. 2 (a). By Fig. 2 (b), one arrives at that the control input signals are within $[-80,50]$. Fig. 3 proves the effectiveness of the proposed disturbance observer, i.e., $\hat{x}_{3}$ asymptotically converges to the external disturbance after a short transient state.

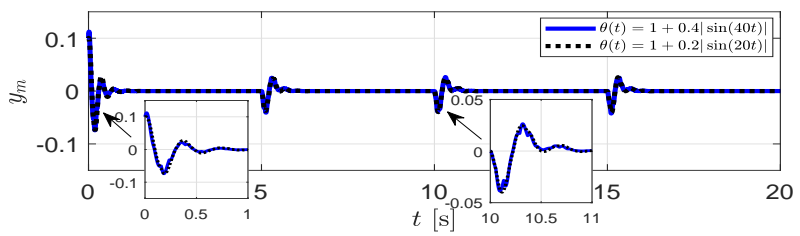

(a)

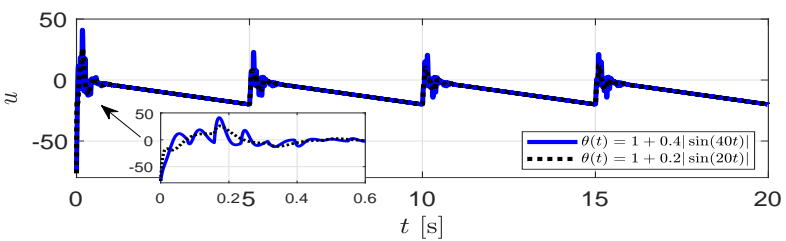

(b)
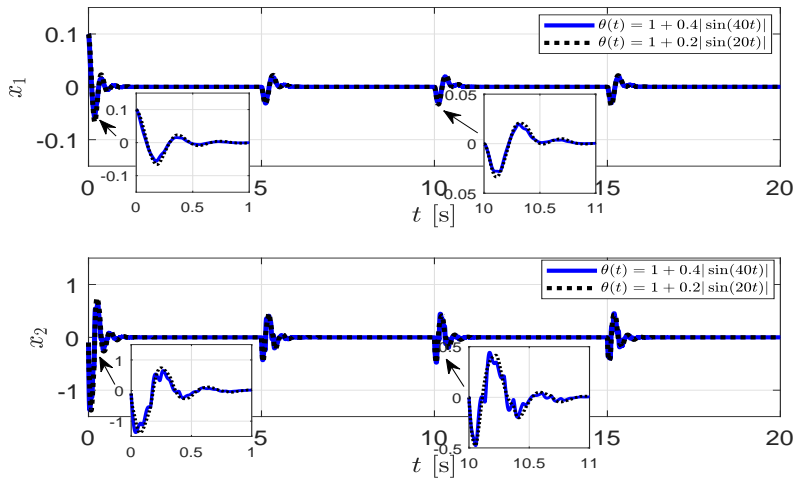

(c)

Figure 2. Control performance. (a) Measured output. (b) Control input. (c) System states.

\section{Application to Angle Control of a Helicopter Model}

In this section, both the feasibility and effectiveness of the proposed DOBC method are illustrated by the application to control of a helicopter model.

\section{A. System Description and Controller Design}

As shown in Fig. 4(a), the tested helicopter model consists of two direct current (DC) motors which drive the corresponding propellers and provide two degrees of freedom of
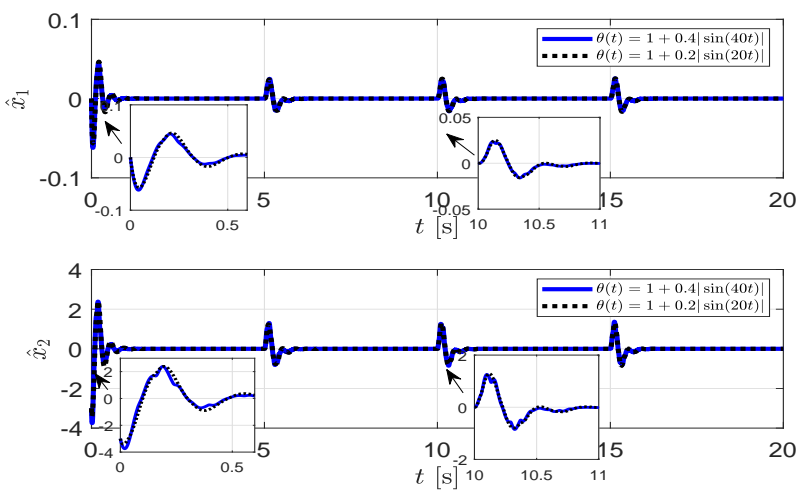

(a)

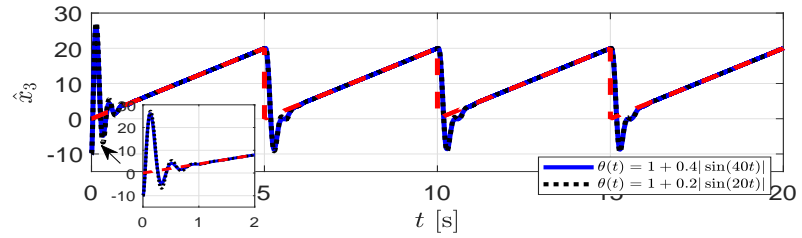

(b)

Figure 3. Estimation performance. (a) Estimated system states. (b) Estimated external disturbance.

motion, i.e., elevation and azimuth. Both angles are measured by incremental rotary encoders. In this paper, we only consider the elevation channel by physically fixing its azimuth one. The measurement data are acquired by a peripheral component interconnect card and are accessible in MATLAB/Simulink environment, from which the torque commands can also be sent to the motor drives. Detailed information on both hardware and software is introduced in [50].

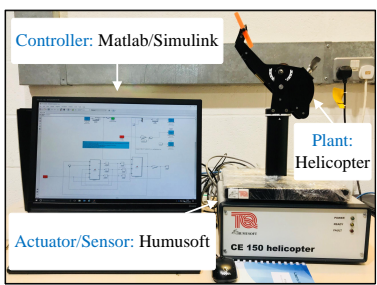

(a)

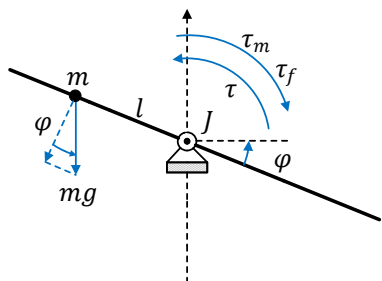

(b)
Figure 4. Helicopter model. (a) Setup. (b) Vertical torque analysis

With the aid of vertical torque analysis in Fig. 4(b), its ideal dynamic model is described as follows:

$$
J \ddot{\varphi}=\tau-\tau_{m}-\tau_{f}
$$

where $J\left(4.37 \times 10^{-3}\left[\mathrm{~kg} \cdot \mathrm{m}^{2}\right]\right)$ is the moment of inertia of the helicopter body around horizontal axis; $\varphi$ [rad] is the elevation angle; $\tau[\mathrm{N} \cdot \mathrm{m}]$ is the elevation driving torque; $\tau_{m}$ $[\mathrm{N} \cdot \mathrm{m}]$ is the gravitational torque, equals $\tau_{g} \cos \varphi, \tau_{g} \triangleq m g l$; and $\tau_{f}[\mathrm{~N} \cdot \mathrm{m}]$ is the friction torque using Stribeck model, equals $\tau_{f}=\tau_{c}+\left(\tau_{s}-\tau_{c}\right) \exp \left(-\dot{\varphi}^{2} / v_{s}^{2}\right)+\tau_{v} \dot{\varphi}, \tau_{c}, \tau_{v}, \tau_{s}$ and $v_{s}$ are the unknown Coulomb parameter, viscous parameter, static friction parameter and the Stribeck rate, respectively.

By first letting $x \triangleq \operatorname{col}(\varphi, \dot{\varphi}), y \triangleq \varphi, u \triangleq \tau / J, d(t) \triangleq$ $-\left(\tau_{s}+\tau_{g}\right) / J, f_{1}(t, x) \triangleq 0, f_{2}(t, x) \triangleq-\tau_{g}\left(\cos x_{1}-1\right) / J-$ $\tau_{v} x_{2} / J-\left(\tau_{s}-\tau_{c}\right)\left(\exp \left(-x_{2}^{2} / v_{s}^{2}\right)-1\right) / J$, system (17) can be 
transformed into the standard one (1). Based upon the standard model, the proposed controller can be then straightforward designed. It is worth pointing out that the practical elevation angle $\varphi$ is within the range of $[-\pi / 3, \pi / 3]$ due to physical constraints, as shown in Fig. 4(a).

\section{B. Experimental Results: Robustness Tests Against Multiple Sensor Errors}

In what follows, both the linear ADRC method [4] and the conventional PID method are used to design the angle controller for comparisons. The considered three controllers are listed as follows:

1) The PID controller: $u=-k_{p} y_{m}-k_{i} \int_{o}^{t} y_{m} d t-k_{d} \dot{y}_{m}$.

2) The linear ADRCer:

$$
\begin{aligned}
& \dot{\hat{x}}_{1}=\hat{x}_{2}-h_{1}\left(\hat{x}_{1}-y_{m}\right), \quad \dot{\hat{x}}_{2}=u+\hat{x}_{3}-h_{2}\left(\hat{x}_{1}-y_{m}\right), \\
& \dot{\hat{x}}_{3}=-h_{3}\left(\hat{x}_{1}-y_{m}\right), u=-k_{2} \hat{x}_{1}-k_{1} \hat{x}_{2}-\hat{x}_{3} .
\end{aligned}
$$

3) The proposed controller:

$$
\begin{aligned}
& \dot{\hat{x}}_{1}=\hat{x}_{2}-h_{1} \hat{x}_{1}, \dot{\hat{x}}_{2}=u+\hat{x}_{3}-h_{2} \hat{x}_{1}, \\
& \dot{\hat{x}}_{3}=-h_{3} \hat{x}_{1}, u=-k_{2} y_{m}-k_{1} \hat{x}_{2}-\hat{x}_{3} .
\end{aligned}
$$

It is worth noting that the linear ADRCer has the similar structure with the proposed controller. However, due to the unknown relationship between $x_{1}$ and $y_{m}$, the estimation error based correction term in the linear ADRCer has to be chosen as $\hat{x}_{1}-y_{m}$ for a compromise, rather than the conventional one, which would deteriorate its control performance. Before experimental verification, efforts have been primarily made in parameter tuning. Following the guidelines in [34], [35], the parameters of the two kinds of disturbance observers are chosen in a practical "trial and error" way, considering a compromise between estimation/stabilization rates and noise amplification. For fair comparisons with the conventional PID method, whose parameters are empirically tuned following [51], the controller gains of the proposed method and linear ADRC method are chosen the same as the proportional and differential gains of the PID method. Parameters of the proposed controller and the linear ADRCer are $\left(h_{1}, h_{2}, h_{3}\right)=$ $(12,48,64)$ and $\left(k_{1}, k_{2}\right)=(2.6,13.5)$. Parameters of the PID controller are $\left(k_{p}, k_{i}, k_{d}\right)=(13.5,10.9,2.6)$ with a low-pass filter $1 /(1+s / 100)$ added on the differentiation term.

To better demonstrate the features of the proposed method, we imitate the applications with relative poor-quality sensors by artificially multiplying additional sensor sensitivities on the measurement of elevation angle whilst the elevation angle measured by incremental rotary encoder is regarded as the truth value, as shown in Fig. 5. Without loss of generality, in following experiments, we choose a class of continuous but non-differentiable functions $\theta(t)=1+a|\sin (f t)|$, where $a$ and $f[\mathrm{rad} / \mathrm{s}]$ is its amplitude and frequency, respectively.

Figs. 6 to 8 show the curves of the measured elevation angles, the elevation angles, the driving torques under the proposed method, the linear ADRC method and the PID method, respectively whilst Figs. 11 and 12 present the corresponding partial enlarged curves of the dynamic states and steady states, respectively. The curves of the estimated system states and the estimated external disturbances of the proposed disturbance

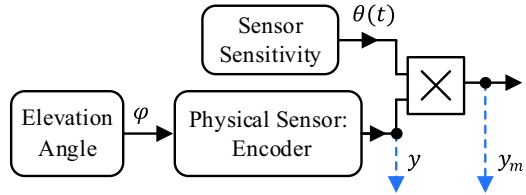

Figure 5. Artificial sensor sensitivity.

observer and the conventional extended observer are presented in Figs. 9 and 10, respectively. Clearly observed from Figs. 6(b), 7(b) and 8(b), one gets that the helicopter model can not move in the beginning. This is because that the driving torque generated by the DC motor is smaller than the sum of gravitational torque and static friction torque at the starting phase, making the helicopter model in the static state. Once adding the artificial sensor sensitivities, the fluctuations exist even in the static states of the measured elevation angles, bringing obstacles to achieve satisfying control performance, as shown in Figs. 6(a), 7(a) and 8(a). In Figs. 9 and 10, the estimated disturbance curves of both the proposed disturbance observer and the conventional extended observer under multiple sensor sensitivities all converge to the same value (i.e., around -460) in the steady states.

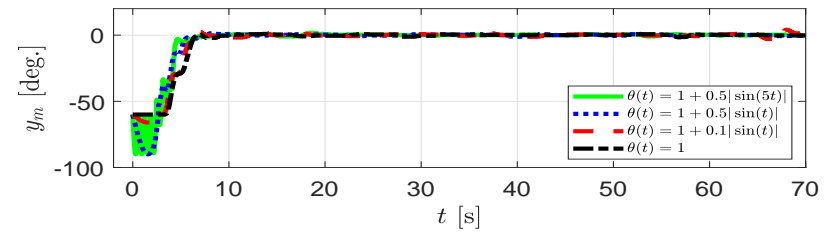

(a)

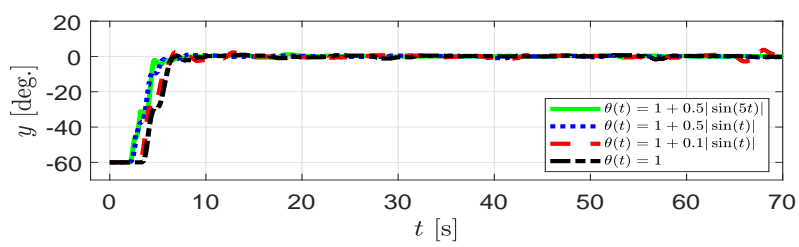

(b)

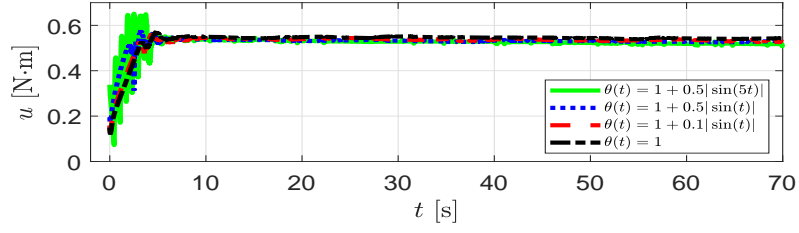

(c)

Figure 6. Robust stabilization performance of the proposed method. (a) Measured elevation angle. (b) Elevation angle. (c) Driving torque.

To make the comparisons clearer and more convincing, the starting time ${ }^{2}$ and settling time ${ }^{3}$ indexes are introduced for the dynamic-state comparisons whilst the integral of squared error $(I S E)^{4}$ index is introduced for the steady-state comparisons. From Table I, one can conclude that compared with both the

\footnotetext{
${ }^{2}$ Time it takes for the angle from the static state to the critical static state. ${ }^{3}$ Time it takes for the angle from the critical static state to the steady state. ${ }^{4} \mathrm{ISE}_{<t_{1}, t_{2}>} \triangleq \int_{t_{1}}^{t_{2}} y(t)^{2} d t$.
} 


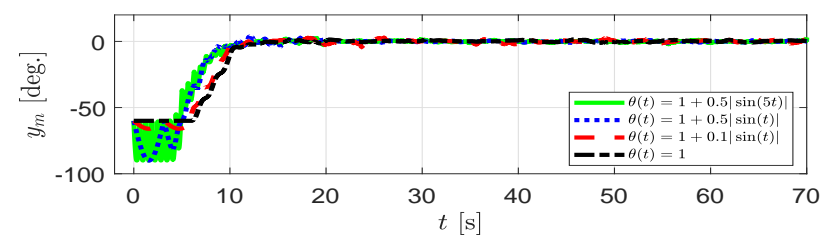

(a)

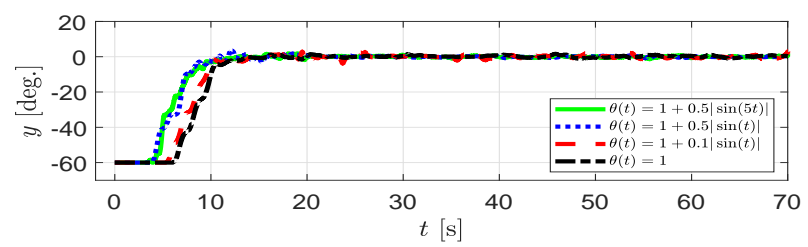

(b)

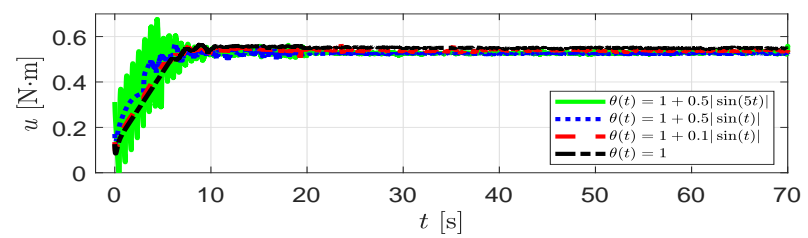

(c)

Figure 7. Robust stabilization performance of the ADRC method. (a) Measured elevation angle. (b) Elevation angle. (c) Driving torque.

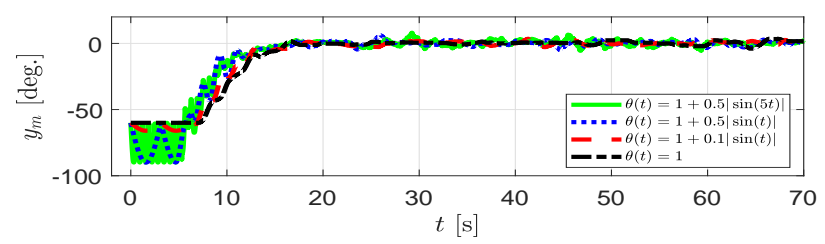

(a)

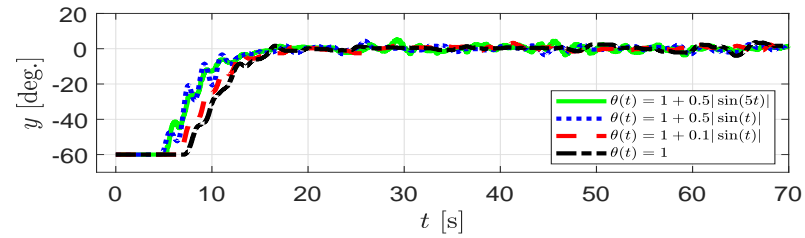

(b)

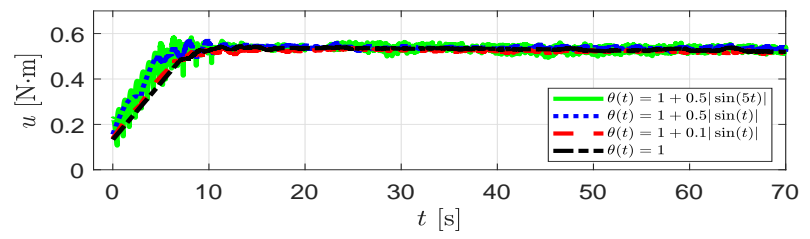

(c)

Figure 8. Robust stabilization performance of the PID method. (a) Measured elevation angle. (b) Elevation angle. (c) Driving torque.

linear ADRC method the PID method, the proposed method possesses faster convergence rate and higher precision.

\section{Conclusion}

This paper has presented a systematic disturbance estimation and attenuation approach for a class of disturbed uncertain nonlinear systems with output measurement errors. A new disturbance observer, which is only explicitly constructed by the control input, has been proposed. Linear feedback
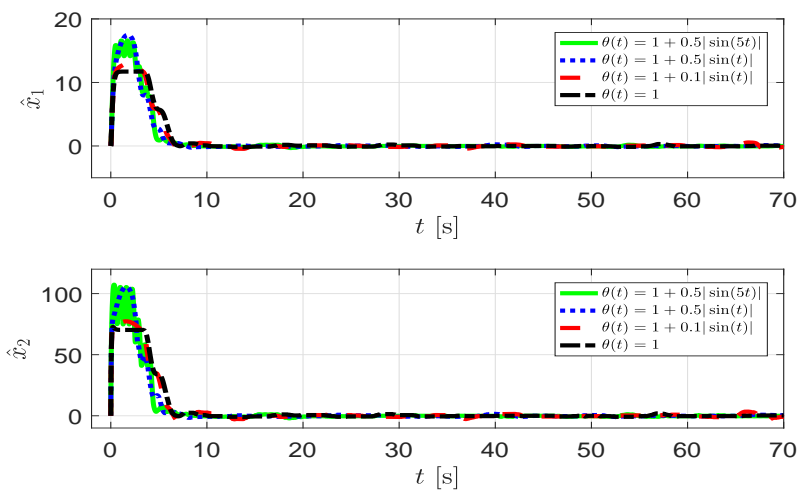

(a)

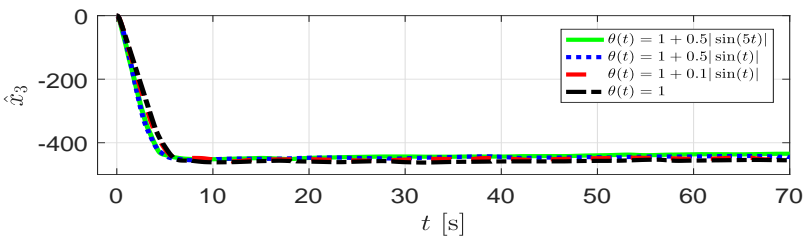

(b)

Figure 9. Estimation performance of the proposed method. (a) Estimated system states. (b) Estimated external disturbance.
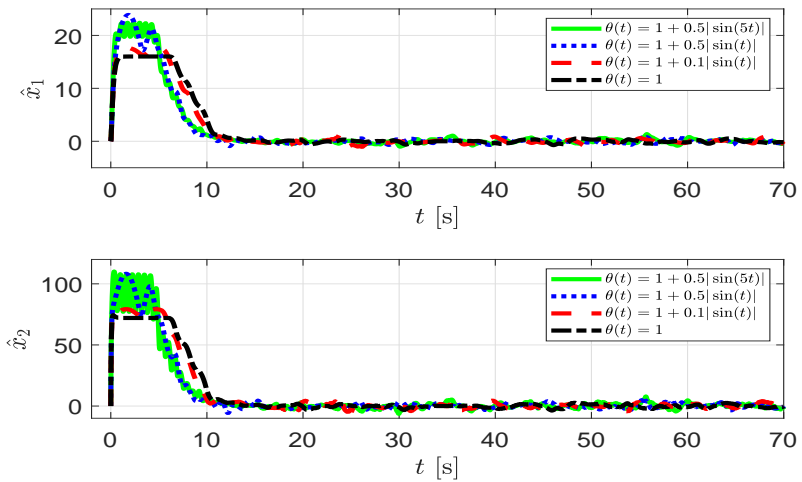

(a)

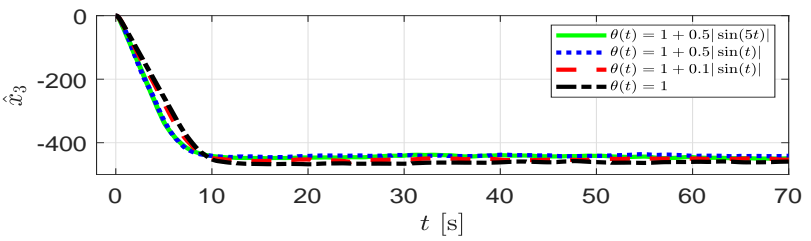

(b)

Figure 10. Estimation performance of the ADRC method. (a) Estimated system states. (b) Estimated external disturbance.

controller with a corresponding feedforward compensation part for the external disturbance has then been designed. It has been proved that the proposed method endows the closed-loop system with strong robustness against both output measurement errors and system uncertainties. The elevation angle stabilization of a helicopter model, as a benchmark, has been conducted to illustrate the feasibility and efficacy of the proposed method. As demonstrated in the experimental results, the proposed method has improved the performance of fast and high-precision stabilization, even with multiple sensor sensitivities. Future work will be concentrated on investigating 

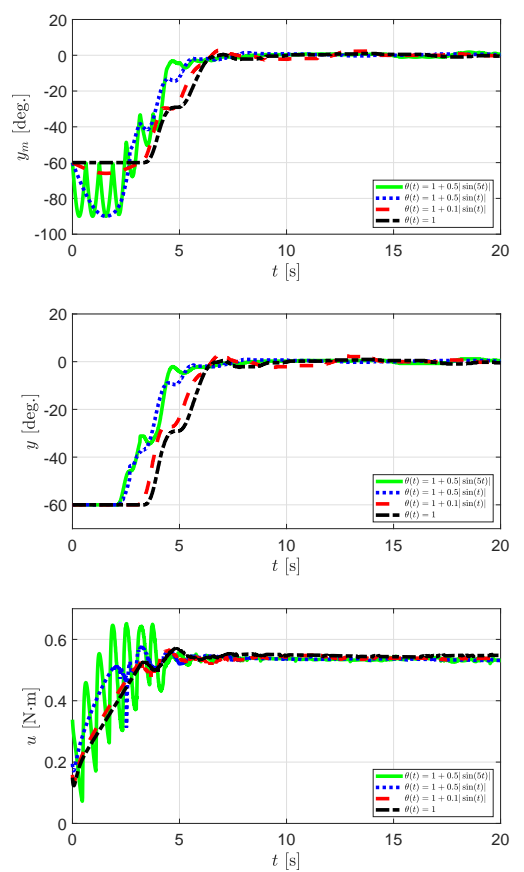

(a)
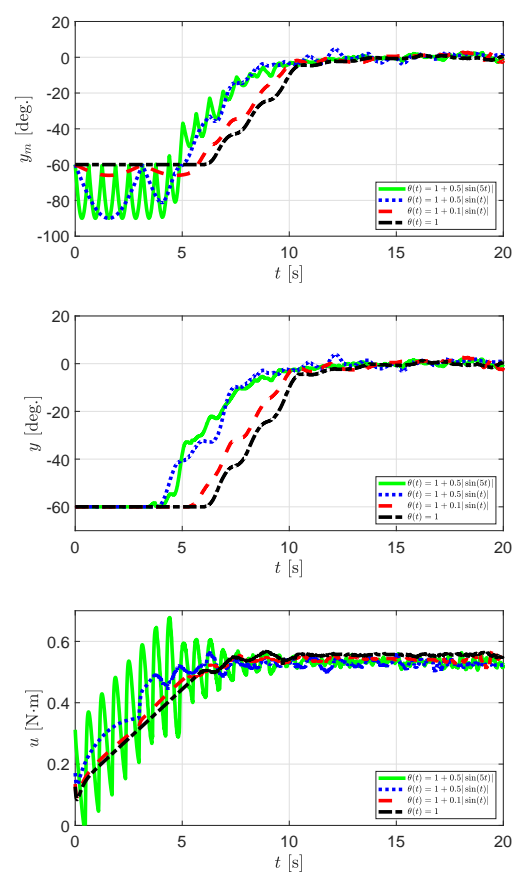

(b)
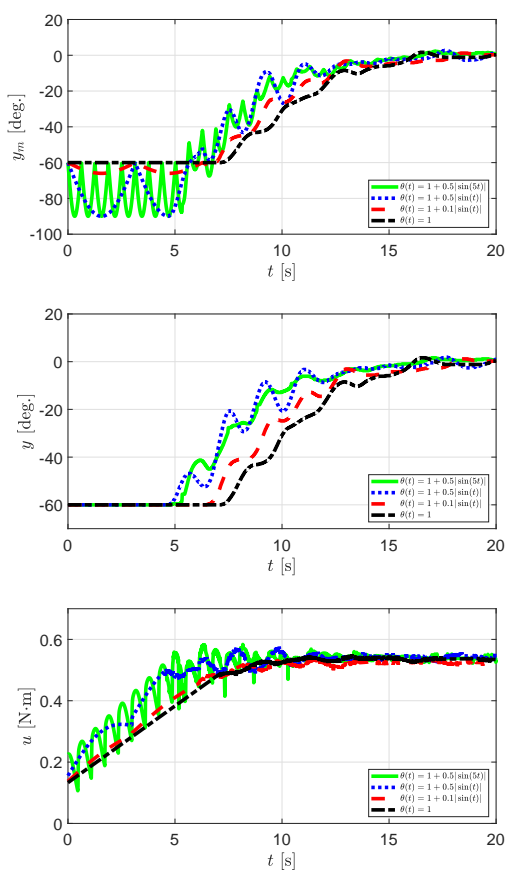

(c)

Figure 11. Partial enlarged dynamic-state curves within [0s, 20s]. (a) The proposed method. (b) The ADRC method. (c) The PID method.

Table I

Indexes of Robustness Tests Against Multiple Sensor Errors.

\begin{tabular}{lllll}
\hline Method & $\begin{array}{l}\text { Sensor } \\
\text { sensitivity }\end{array}$ & $\begin{array}{l}\text { Starting } \\
\text { time }[\mathrm{s}]\end{array}$ & $\begin{array}{l}\text { Settling } \\
\text { time }[\mathrm{s}]\end{array}$ & $\begin{array}{l}\text { ISE } \\
<20 \mathrm{~s}, 70 \mathrm{~s}>\end{array}$ \\
\hline \multirow{3}{*}{ Proposed } & 1 & 3.39 & 6.61 & 10.57 \\
& $1+0.1|\sin (t)|$ & 3.07 & 5.87 & 38.59 \\
& $1+0.5|\sin (t)|$ & 2.09 & 10.71 & 6.17 \\
& $1+0.5|\sin (5 t)|$ & 2.12 & 7.07 & 2.41 \\
\hline \multirow{3}{*}{ ADRC } & 1 & 6.06 & 8.49 & 14.29 \\
& $1+0.1|\sin (t)|$ & 5.32 & 7.19 & 51.79 \\
& $1+0.5|\sin (t)|$ & 3.84 & 11.26 & 8.09 \\
& $1+0.5|\sin (5 t)|$ & 3.98 & 10.90 & 11.47 \\
\hline \multirow{3}{*}{ PID } & 1 & 7.19 & 9.84 & 85.24 \\
& $1+0.1|\sin (t)|$ & 6.49 & 12.83 & 77.98 \\
& $1+0.5|\sin (t)|$ & 4.69 & 13.28 & 87.72 \\
& $1+0.5|\sin (5 t)|$ & 5.17 & 11.65 & 109.87 \\
\hline
\end{tabular}

and attenuating the adverse effect of noise under the proposed control structure.

\section{APPENDIX}

Appendix A collects the definitions of semiglobal stability and semiglobal practical stability whilst Appendixes B to D collect the proofs of Propositions 1 to 3.

\section{A. Definitions}

Consider the following system:

$$
\dot{x}=f(x)+g(x) u, x \in \mathbb{R}^{n}
$$

with $x=0$ as its equilibrium.
1) Semiglobal Stability [39, Chap. 9.3]: System (18) is said to be semiglobally stabilizable if, for each (arbitrarily large) compact subset $\mathbb{P} \subset \mathbb{R}^{n}$, there exists a feedback law $u=u(x)$, which in general depends on $\mathbb{P}$, such that the equilibrium is locally asymptotically stable and $x(0) \in \mathbb{P} \Rightarrow x(\infty)=0$.

2) Semiglobal Practical Stability [40, Chap. 12.1]: System (18) is said to be semiglobally practically stabilizable if, for any (arbitrarily large) compact subset $\mathbb{P} \subset \mathbb{R}^{n}$ and any arbitrarily small compact subset $\mathbb{Q} \subset \mathbb{R}^{n}$, there exists a feedback law $u=u(x)$, which in general depends on both $\mathbb{P}$ and $\mathbb{Q}$, such that any trajectory with initial condition in $\mathbb{P}$ is captured by $\mathbb{Q}$.

\section{B. Proof of Proposition 1}

Proof: By Assumption 2, we have

$$
\begin{aligned}
\left|\frac{f_{i}(t, x)}{\ell_{o}^{i-1}}\right| \leq & \frac{g_{i}(y)}{\ell_{o}^{i-1}}\left(\left|\tilde{x}_{1}\right|+\cdots+\left|\left(\ell_{o} \ell_{c}\right)^{i-1} \tilde{x}_{i}+\ell_{o}^{i-1} \tilde{e}_{i}\right|\right) \\
\leq & g_{i}(y) \ell_{c}^{n-1}\left(\left|\tilde{x}_{1}\right|+\cdots+\left|\tilde{x}_{i}\right|+\cdots+\left|\tilde{x}_{n}\right|\right) \\
& +g_{i}(y)\left(\left|\tilde{e}_{1}\right|+\cdots+\left|\tilde{e}_{i}\right|+\cdots\left|\tilde{e}_{n+m}\right|\right) \\
\leq & g(y)\left(\ell_{c}^{n-1}\|\tilde{x}\|+\|\tilde{e}\|\right), \quad i \in \mathbb{N}_{1: n}
\end{aligned}
$$

where $g(y) \triangleq \sqrt{n+m} \cdot \max \left\{g_{1}(y), \cdots, g_{n}(y)\right\}$. Besides, one gets

$$
\left|f_{1}(t, x)\right| \leq g_{1}(y)\left|x_{1}\right| \leq g(y)\|\tilde{x}\|
$$

By Assumption 3, (19) gives

$$
\|F\|^{2} \leq 2 n g^{2}(y)\left(\ell_{c}^{2 n-2}\|\tilde{x}\|^{2}+\|\tilde{e}\|^{2}\right)+\frac{\bar{r}^{2}}{\ell_{o}^{2(n+m-1)}} .
$$



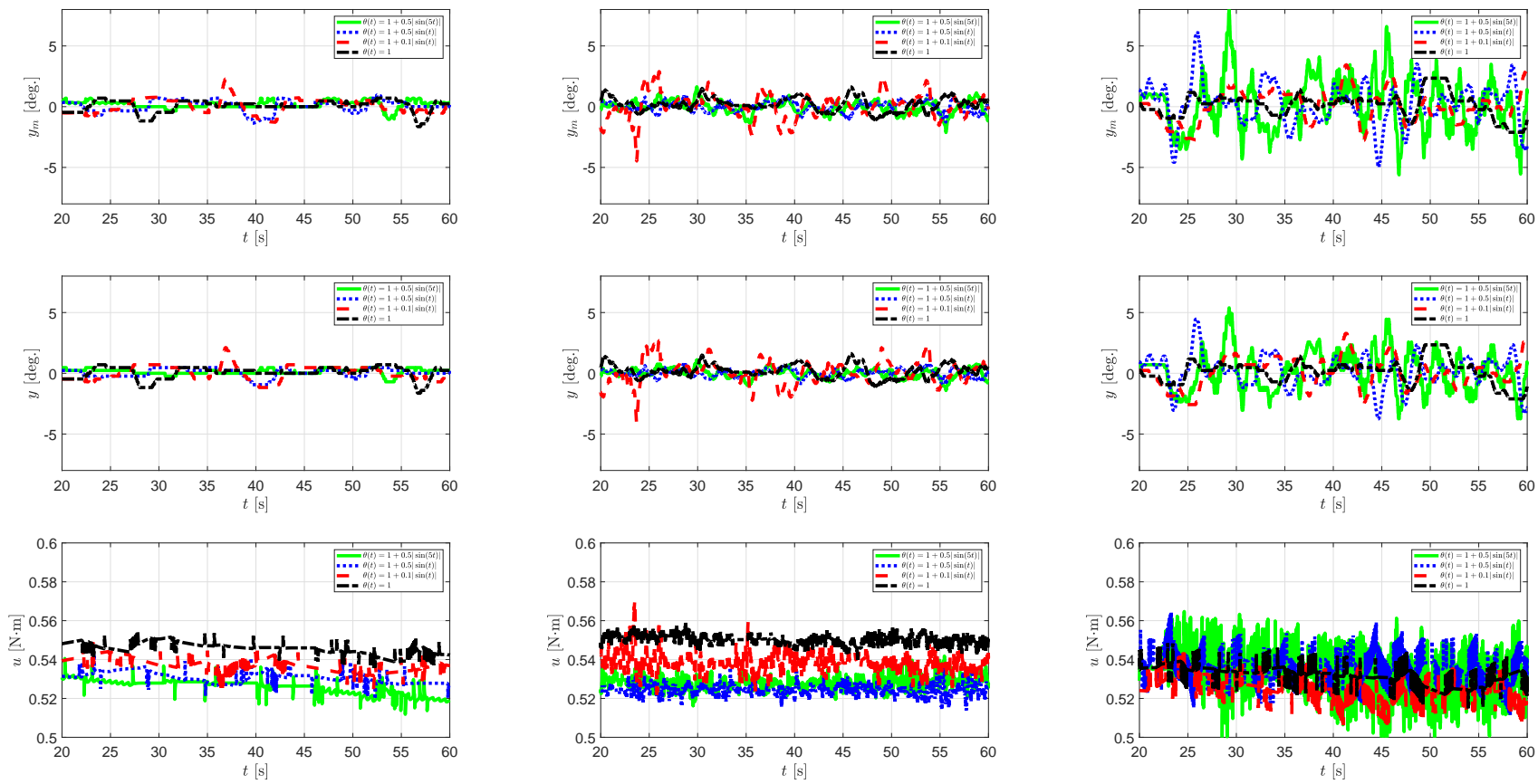

(a)

(b)

(c)

Figure 12. Partial enlarged steady-state curves within [20s, 60s]. (a) The proposed method. (b) The ADRC method. (c) The PID method.

With the assist of (21), one gets the following inequation:

$$
\begin{aligned}
\left.\dot{V}_{o}(\tilde{e})\right|_{(6)}=-\ell_{o}\|\tilde{e}\|^{2}+2 \tilde{e}^{\top} P_{o} F+2 \ell_{o} \tilde{e}^{\top} P_{o} H_{o} \tilde{x}_{1} \\
\leq-\ell_{o}\|\tilde{e}\|^{2}+2 \lambda_{o}\|\tilde{e}\|\|F\|+2 \ell_{o} \lambda_{o}\left\|H_{o}\right\|\|\tilde{e}\|\|\tilde{x}\| \\
\leq-\ell_{o}\|\tilde{e}\|^{2}+\lambda_{o}^{2}\|\tilde{e}\|^{2}+2 n g^{2}(y)\left(\ell_{c}^{2 n-2}\|\tilde{x}\|^{2}+\|\tilde{e}\|^{2}\right)+\frac{\ell_{o}}{2}\|\tilde{e}\|^{2} \\
\quad+2 \ell_{o} \lambda_{o}^{2}\left\|H_{o}\right\|^{2}\|\tilde{x}\|^{2}+\frac{\bar{r}^{2}}{\ell_{o}^{2(n+m-1)}} \\
=-\left(\frac{\ell_{o}}{2}-c_{1}-2 n g^{2}(y)\right)\|\tilde{e}\|^{2}+\left(c_{2} \ell_{o}+2 n g^{2}(y) \ell_{c}^{2 n-2}\right)\|\tilde{x}\|^{2} \\
\quad+\frac{\bar{r}^{2}}{\ell_{o}^{2(n+m-1)}}
\end{aligned}
$$

where $\lambda_{o} \triangleq \lambda_{\text {max }}\left(P_{o}\right), c_{1} \triangleq \lambda_{o}^{2}$ and $c_{2} \triangleq 2 \lambda_{o}^{2}\left\|H_{o}\right\|^{2}$.

This completes the proof of Proposition 1 .

\section{Proof of Proposition 2}

Proof: With (20) in mind, the following inequation holds:

$$
\begin{aligned}
& \left.\dot{V}_{c}(\tilde{x})\right|_{(8)}=-\ell_{o} \ell_{c}\|\tilde{x}\|^{2}+2 \frac{\ell_{o}}{\ell_{c}} \tilde{x}^{\top} P_{c} H_{c}\left(\tilde{x}_{1}-\tilde{e}_{1}\right) \\
& \quad+2 \tilde{x}^{\top} P_{c} I_{1}\left(\ell_{o} \tilde{e}_{2}+f_{1}(t, x)\right)+2 \ell_{o} \ell_{c} \tilde{k}_{n}(1-\theta(t)) \tilde{x}^{\top} P_{c} I_{2} \tilde{x}_{1} \\
& \leq-c^{*} \ell_{o} \ell_{c}\|\tilde{x}\|^{2}+2 \lambda_{c}\|\tilde{x}\|\left(\left(c_{3} \frac{\ell_{o}}{\ell_{c}}+\ell_{o}\right)\|\tilde{e}\|+\left(c_{3} \frac{\ell_{o}}{\ell_{c}}+g(y)\right)\|\tilde{x}\|\right) \\
& \leq-c^{*} \ell_{o} \ell_{c}\|\tilde{x}\|^{2}+2 \lambda_{c} c_{4} \ell_{o}\|\tilde{x}\|\|\tilde{e}\|+2 \lambda_{c}\left(c_{3} \ell_{o}+g(y)\right)\|\tilde{x}\|^{2} \\
& \leq-c^{*} \ell_{o} \ell_{c}\|\tilde{x}\|^{2}+4 \lambda_{c}^{2} c_{4}^{2} \ell_{o}\|\tilde{x}\|^{2}+\frac{\ell_{o}}{4}\|\tilde{e}\|^{2}+2 \lambda_{c}\left(c_{3} \ell_{o}+g(y)\right)\|\tilde{x}\|^{2} \\
& \leq-c^{*} \ell_{o} \ell_{c}\|\tilde{x}\|^{2}+\left(c_{5} \ell_{o}+2 \lambda_{c} g(y)\right)\|\tilde{x}\|^{2}+\frac{\ell_{o}}{4}\|\tilde{e}\|^{2}
\end{aligned}
$$

where $\lambda_{c} \triangleq \lambda_{\max }\left(P_{c}\right), c_{3} \triangleq \sqrt{\tilde{h}_{2}^{2}+\cdots+\tilde{h}_{n}^{2}}, c_{4} \triangleq 1+c_{3}, c_{5} \triangleq$ $4 \lambda_{c}^{2} c_{4}^{2}+2 \lambda_{c} c_{3}, c^{*} \triangleq 1-2 \tilde{k}_{n} \lambda_{c} \Delta>0$ and

$$
0 \leq \Delta<\min \left\{1, \frac{1}{2 \tilde{k}_{n} \lambda_{c}}\right\} \text {. }
$$

This completes the proof of Proposition 2.

\section{Proof of Proposition 3}

Proof: Denote $V(t) \triangleq V(\tilde{e}(t), \tilde{x}(t))$. If Proposition 3 is not true, there must exists a time instant $t^{*}>0$ such that $V\left(t^{*}\right)>$ $M$. The following two cases with regard to different $V(0)$ will be discussed. Before the discussion, remind that $V(0) \leq M$ holds due to the definition of $M$ in (13).

1) Case $I-V(0)<M$ : In Case I, there must exist time instants $t_{1}$ and $t_{2}, t_{1} \in\left(0, t_{2}\right)$ such that the following three inferences hold:

$\left(B_{1}\right) \quad V(t) \in[0, M)$ holds for $t \in\left[0, t_{1}\right)$.

(B) $V\left(t_{1}\right)=M$.

(B) $V(t) \in(M, \infty)$ holds for $t \in\left(t_{1}, t_{2}\right]$.

Inferences $\left(B_{1}\right)$ and $\left(B_{2}\right)$ will result in the following one:

$\left(B_{4}\right)$ There must exists a time instant $t_{3}, t_{3} \in\left[0, t_{1}\right)$ such that $V(t) \in[M / 2, M)$ holds for $t \in\left[t_{3}, t_{1}\right)$.

Noting that $\ell_{o} \geq\left(c_{6} C M /\left(4 \bar{r}^{2}\right)\right)^{2 n+2 m-2}$ and (16) still holds for $t \in\left[t_{3}, t_{1}\right]$, one arrives at that $\left.\dot{V}(t)\right|_{t \in\left[t_{3}, t_{1}\right]} \leq-c_{6} C M / 4<0$, which means that $V\left(t_{3}\right)>V\left(t_{1}\right)=M$. This leads to a contradiction and implies that Proposition 3 holds [see Fig. 13(a)].

2) Case $I I-V(0)=M$ : Noting that $\dot{V}(0) \leq-3 c_{6} C M / 4<0$ and $V(t) \in \mathbb{C}^{0}$, there must exists a time instant $t_{4}>0$ such that $V\left(t_{4}\right)<M$. After $t_{4}$, the proof is analogous [see Fig. 13(b)].

This completes the proof of Proposition 3. 


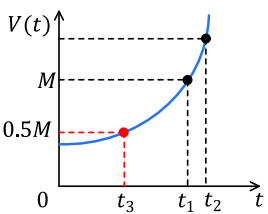

(a)

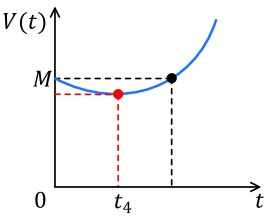

(b)
Figure 13. Case studies of $V(t)$. (a) Case I. (b) Case II.

\section{References}

[1] K. J. Åström and P. R. Kumar, "Control: A perspective," Automatica, vol. 50, no. 1, pp. 3-43, Dec. 2014.

[2] K. Ohishi, K. Ohnishi, and K. Miyachi, "Torque-speed regulation of DC motor based on load torque estimation," in Proc. JIEE/Int. Power Electron. Conf., Tokyo, Japan, Mar. 1983, pp. 1209-1216.

[3] W.-H. Chen, "Disturbance observer based control for nonlinear systems," IEEE/ASME Trans. Mechatron., vol. 9, no. 4, pp. 706-710, Dec. 2004.

[4] J. Han, "From PID to active disturbance rejection control," IEEE Trans. Ind. Electron., vol. 56, no. 3, pp. 900-906, Mar. 2009.

[5] L. Guo and S. Cao, "Anti-disturbance control theory for systems with multiple disturbances: A survey,” ISA Trans., vol. 53, no. 4, pp. 846-849, Oct. 2014.

[6] R. Madoński, M. Kordasz, and P. Sauer, "Application of a disturbancerejection controller for robotic-enhanced limb rehabilitation trainings," ISA Trans., vol. 53, no. 4, pp. 899-908, Oct. 2014.

[7] H. Yu, S. Huang, G. Chen, Y. Pan, and Z. Guo, "Human-robot interaction control of rehabilitation robots with series elastic actuators," IEEE Trans. Robot., vol. 31, no. 5, pp. 1089-1100, Oct. 2015.

[8] N. Sun, T. Yang, Y. Fang, B. Lu, and Y. Qian, "Nonlinear motion control of underactuated three-dimensional boom cranes with hardware experiments," IEEE Trans. Ind. Informat., vol. 14, no. 3, pp. 887-897, Mar. 2018.

[9] M. Ruderman, A. Ruderman, and T. Bertram, "Observer-based compensation of additive periodic torque disturbances in permanent magnet motors," IEEE Trans. Ind. Informat., vol. 9, no. 2, pp. 1130-1138, May. 2013.

[10] S. Zheng, B. Han, and L. Guo, "Composite hierarchical antidisturbance control for magnetic bearing system subject to multiple external disturbances," IEEE Trans. Ind. Electron., vol. 61, no. 12, pp. 7004-7012, Dec. 2014.

[11] J. Yang, W.-H. Chen, S. Li, L. Guo, and Y. Yan, "Disturbance/uncertainty estimation and attenuation techniques in PMSM drives-A survey," IEEE Trans. Ind. Electron., vol. 64, no. 4, pp. 3273-3285, Apr. 2017.

[12] J. Wang, F. Wang, G. Wang, S. Li, and L. Yu, "Generalized proportional integral observer-based robust finite control set predictive current control for induction motor systems with time-varying disturbances," IEEE Trans. Ind. Informat., vol. 4, no. 9, pp. 4159-4168, Sep. 2018.

[13] J. Yang, H. Cui, S. Li, and A. Zolotas, "Optimized active disturbance rejection control for DC-DC buck converters with uncertainties using a reduced-order GPI observer," IEEE Trans. Circuits Syst. I-Regul. Pap. vol. 65, no. 2, pp. 832-841, Jul. 2018.

[14] R. Errouissi, A. Al-Durra, and S. M. Muyeen, "Design and implementation of a nonlinear PI predictive controller for a grid-tied photovoltaic inverter," IEEE Trans. Ind. Electron., vol. 64, no. 2, pp. 1241-1249, Feb. 2017.

[15] Y. Joo, G. Park, J. Back, and H. Shim, "Embedding internal model in disturbance observer with robust stability," IEEE Trans. Autom. Control, vol. 61, no. 10, pp. 3128-3133, Oct. 2016

[16] K.-S. Kim, K.-H. Rew, and S. Kim, "Disturbance observer for estimating higher order disturbances in time series expansion," IEEE Trans. Autom. Control, vol. 55, no. 8, pp. 1905-1911, Aug. 2010.

[17] H. Sira-Ramirez, A. Luviano-Juárez, M. Ramírez-Neria, and E. W. Zurita-Bustamante, Active Disturbance Rejection Control of Dynamic Systems: A Flatness Based Approach. Kidlington, U.K.: ButterworthHeinemann, 2017.

[18] W.-H. Chen, "Harmonic disturbance observer for nonlinear systems," $J$ Dyn. Syst. Meas. Control, vol. 125, no. 1, pp. 114-117, Mar. 2003.

[19] H. Muramatsu and S. Katsura, "Adaptive periodic-disturbance observer for periodic-disturbance suppression," IEEE Trans. Ind. Informat., vol. 14 , no. 10 , pp. 4446-4456, Oct. 2018.
[20] Y. Yan, J. Yang, Z. Sun, C. Zhang, S. Li, and H. Yu, "Robust speed regulation for PMSM servo system with multiple sources of disturbances via an augmented disturbance observer," IEEE/ASME Trans. Mechatron., vol. 23, no. 2, pp. 769-780, Apr. 2018.

[21] A. Levant, "Higher-order sliding modes, differentiation and outputfeedback control," Int. J. Control, vol. 76, no. 9-10, pp. 924-941, Nov. 2003.

[22] J. Yang, J. Su, S. Li, and X. Yu, "High-order mismatched disturbance compensation for motion control systems via a continuous dynamic sliding-mode approach," IEEE Trans. Ind. Informat., vol. 10, no. 1, pp. 604-614, Feb. 2014.

[23] S. Li, H. Sun, J. Yang, and X. Yu, "Continuous finite-time output regulation for disturbed systems under mismatching condition," IEEE Trans. Autom. Control, vol. 60, no. 1, pp. 277-282, Jan. 2015.

[24] C. Zhang, Y. Yan, C. Wen, J. Yang, and H. Yu, "A nonsmooth composite control design framework for nonlinear systems with mismatched disturbances: Algorithms and experimental tests," IEEE Trans. Ind. Electron., vol. 65, no. 11, pp. 8828-8839, Nov 2018 .

[25] J. Sun, J. Yang, S. Li, and W. X. Zheng, "Sampled-data-based eventtriggered active disturbance rejection control for disturbed systems in networked environment," IEEE Trans. Cybern., vol. 49, no. 2, pp. 556566, Feb. 2019.

[26] Y. Huang, J. Wang, D. Shi, and L. Shi, "Towards event-triggered extended state observer," IEEE Trans. Autom. Control, vol. 63, no. 6, pp. 1842-1849, Jun. 2018.

[27] N. H. Jo, C. Jeon, and H. Shim, "Noise reduction disturbance observer for disturbance attenuation and noise suppression," IEEE Trans. Ind Electron., vol. 64, no. 2, pp. 1381-1391, Feb. 2017.

[28] W. Xue, W. Bai, S. Yang, K. Song, Y. Huang, and H. Xie, "ADRC with adaptive extended state observer and its application to air-fuel ratio control in gasoline engines," IEEE Trans. Ind. Electron., vol. 62, no. 9, pp. 5847-5857, Sep. 2015.

[29] B. Xu, F. Sun, Y. Pan, and B. Chen, "Disturbance observer based composite learning fuzzy control of nonlinear systems with unknown dead zone," IEEE Trans. Syst. Man Cybern. -Syst., vol. 47, no. 8, pp. 1854-1862, Aug. 2017.

[30] J. Yao and W. Deng, "Active disturbance rejection adaptive control of uncertain nonlinear systems: Theory and application," Nonlinear Dyn., vol. 89, no. 3, pp. 1611-1624, May. 2017.

[31] J. Taylor, Introduction to Error Analysis, the Study of Uncertainties in Physical Measurements. New York, USA: University Science Books, 1997.

[32] T. Endo, M. Koyanagi, and A. Nakamura, "High-accuracy josephson potentiometer," IEEE Trans. Instrum. Meas., vol. 32, no. 1, pp. 267271, Mar. 1983

[33] O. Bourquardez, R. Mahony, N. Guenard, F. Chaumette, T. Hamel, and L. Eck, "Image-based visual servo control of the translation kinematics of a quadrotor aerial vehicle," IEEE Trans. Robot., vol. 25, no. 3, pp. 743-749, Jun. 2009.

[34] Z. Gao, "Scaling and bandwidth-parameterization based controller tuning," in Proc. Amer. Control Conf. (ACC), Denver, Colorado, Jun. 2003 pp. 4989-4996.

[35] Q. Zheng, L. Q. Gao, and Z. Gao, "On stability analysis of active disturbance rejection control for nonlinear time-varying plants with unknown dynamics," in Proc. IEEE Conf. Decis. Control (CDC), New Orleans, LA, USA, Dec. 2007, pp. 3501-3506.

[36] Y. Huang and W. Xue, "Active disturbance rejection control: Methodology and theoretical analysis," ISA Trans., vol. 53, no. 4, pp. 963-976, Apr. 2014.

[37] J. Zhai and C. Qian, "Global control of nonlinear systems with uncertain output function using homogeneous domination approach," Int. J. Robust Nonlinear Control, vol. 22, no. 14, pp. 1543-1561, Jul. 2012

[38] C.-C. Chen, C. Qian, Z.-Y. Sun, and Y.-W. Liang, "Global output feedback stabilization of a class of nonlinear systems with unknown measurement sensitivity," IEEE Trans. Autom. Control, vol. 63, no. 7, pp. 2212-2217, Jul. 2018.

[39] A. Isidori, Nonlinear Control Systems (Communications and Control Engineering). London, U.K.: Springer-Verlag, 1995.

[40] - Nonlinear Control Systems II (Communications and Control Engineering). London, U.K.: Springer-Verlag, 1999.

[41] C. Qian, "Semi-global stabilization of a class of uncertain nonlinear systems by linear output feedback," IEEE Trans. Circuits Syst. II, Exp. Briefs, vol. 52, no. 4, pp. 218-222, Apr. 2005.

[42] R. H. B. Exell. Random vs systematic error. [Online]. Available: https://www.physics.umd.edu/courses/Phys276/Hill/Information/Notes /ErrorAnalysis.html 
[43] J. H. Ahrens and H. K. Khalil, "High-gain observers in the presence of measurement noise: A switched-gain approach," Automatica, vol. 45, no. 4, pp. 936-943, Jan. 2009.

[44] A. A. Prasov and H. K. Khalil, "A nonlinear high-gain observer for systems with measurement noise in a feedback control framework," IEEE Trans. Autom. Control, vol. 58, no. 3, pp. 569-580, Mar. 2013.

[45] R. G. Sanfelice and L. Praly, "On the performance of high-gain observers with gain adaptation under measurement noise," Automatica, vol. 47, no. 10 , pp. 2165-2176, Sep. 2011.

[46] A. Poznyak, "Stochastic output noise effects in sliding mode state estimation," Int. J. Control, vol. 76, no. 9-10, pp. 986-999, Nov. 2003.

[47] L. Fraguela, M. T. Angulo, J. A. Moreno, and L. Fridman, "Design of a prescribed convergence time uniform robust exact observer in the presence of measurement noise," in Proc. IEEE Conf. Decis. Control (CDC), Maui, Hawaii, USA., Dec. 2012, pp. 6615-6620.

[48] L. Guo and W.-H. Chen, "Disturbance attenuation and rejection for systems with nonlinearity via DOBC approach," Int. J. Robust Nonlinear Control, vol. 15, no. 3, pp. 109-125, Dec. 2005.

[49] X. Wei and L. Guo, "Composite disturbance-observer-based control and $\mathrm{H} \infty$ control for complex continuous models," Int. J. Robust Nonlinear Control, vol. 20, no. 1, pp. 106-118, Apr. 2010.

[50] C. Humusoft, "150 helicopter model: User's manual," Humusoft, Prague, 2002.

[51] K. H. Ang, G. Chong, and Y. Li, "PID control system analysis, design, and technology," IEEE Trans. Control Syst. Technol., vol. 13, no. 4, pp. 559-576, Jul. 2005.

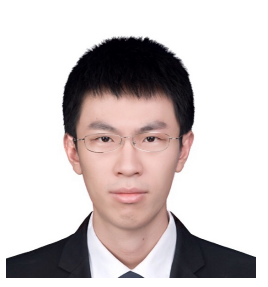

Yunda Yan (S'15-M'18) received the B.Sc degree from School of Automation in Southeast University, Nanjing, China in 2013. He is currently working toward the Ph.D. degree in control theory and control engineering from School of Automation in Southeast University under the guidance of Profs. Shihua Li and Jun Yang. He was a visiting student at Department of Biomedical Engineering, National University of Singapore under the guidance of Prof. Haoyong Yu from Oct. 2016 to Oct. 2017; and was a visiting student at Department of Aeronautical and Automotive Engineering, Loughborough University under the guidance of Dr. Cunjia Liu and Prof. Wen-Hua Chen from Dec. 2017 to Dec. 2018.

His current research interests include the development of predictive control methods, dynamic high-gain control methods, control allocation methods and disturbance modeling and estimation approaches and their applications in motion control systems. He is a member of IEEE.

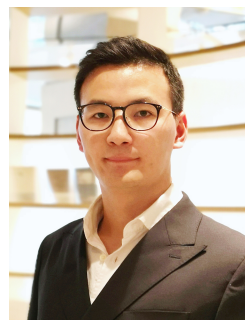

Chuanlin Zhang (M'14) received the B.S. degree in mathematics and the Ph.D. degree in control theory and control engineering from the School of Automation, Southeast University, Nanjing, China, in 2008 and 2014, respectively. He was a Visiting Ph.D. Student with the Department of Electrical and Computer Engineering, University of Texas at San Antonio, USA, from 2011 to 2012; a Visiting Scholar with the Energy Research Institute, Nanyang Technological University, Singapore, from 2016 to 2017; a visiting scholar with Advanced Robotics Center, National University of Singapore, from 2017 to 2018. Since 2014, he has been with the College of Automation Engineering, Shanghai University of Electric Power, Shanghai, where he is currently a Professor.

$\mathrm{He}$ is the principal investigator of several research projects, including Eastern Scholar Program at Shanghai, Leading Talent Program of Shanghai Science and Technology Commission, Chenguang Program by the Shanghai Municipal Education Commission, etc. His research interests include nonlinear system control theory and applications for power systems.

Dr. Zhang serves as a guest editor for Energies. He was the recipient of the Best Poster Paper Award from the 3rd International Federation of Automatic Control International Conference on Intelligent Control and Automation Science (2013).

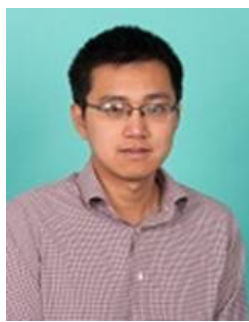

Cunjia Liu (M16) received his B.Eng. and M.Sc. degrees in guidance, navigation, and control from Beihang University, Beijing, China, in 2005 and 2008, respectively. In 2011, he received a Ph.D. in autonomous vehicle control from Loughborough University, Loughborough, Leicestershire, UK. He was a research associate with the Department of Aeronautical and Automotive Engineering at Loughborough University, where he was appointed as a Lecturer and then a Senior Lecturer in Unmanned Vehicles in 2013 and 2018, respectively.

His current research interests include optimization-based control, disturbance-observer-based control, Bayesian information fusion and their applications to autonomous vehicles for flight control, path planning, decision making and situation awareness.

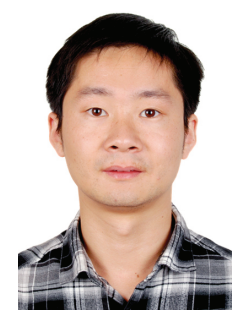

Jun Yang (SM'18) received the B.Sc. degree from the Department of Automatic Control, Northeastern University, Shenyang, China, in 2006, and the Ph.D. degree in control theory and control engineering from the School of Automation, Southeast University, Nanjing, China, in 2011. He is currently a Professor with the School of Automation, Southeast University.

His current research interests include disturbance estimation and compensation, advanced control theory, and its application to flight control systems and motion control systems. Dr. Yang is an Associate Editor of the TRANSACTIONS OF THE INSTITUTE OF MEASUREMENT AND CONTROL. He recieved the ICI prize for best paper of TRANSACTIONS OF THE INSTITUTE OF MEASUREMENT AND CONTROL in 2016.

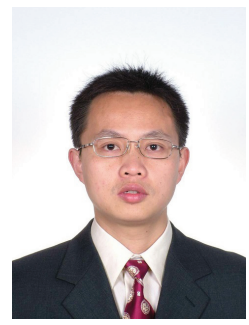

Shihua Li (M'05-SM'10) was born in Pingxiang, Jiangxi Province, China in 1975. He received his bachelor, master, Ph.D. degrees all in Automatic Control from Southeast University, Nanjing, China in 1995, 1998 and 2001, respectively. Since 2001, he has been with School of Automation, Southeast University, where he is currently a professor and the director of Mechatronic Systems Control Laboratory. He has authored or coauthored over 200 technical papers and two books.

His main research interests lie in modeling, analysis and nonlinear control theory with applications to mechatronic systems. Prof. Li is the chairman of IEEE IES Nanjing Chapter. He serves as associate editors or editors of IEEE Transactions on Industrial Electronics, International Journal of Robust \& Nonlinear Control, IET Power Electronics, International Journal of Control, Automation and Systems, International Journal of Electronics and guest editors of IEEE Transactions on Industrial Electronics, International Journal of Robust \& Nonlinear Control and IET Control Theory \& Applications. 